\title{
Antifouling Zwitterion Embedded Forward Osmosis Thin Film Composite Membrane for Highly Concentrated Oily Wastewater Treatment
}

\author{
W.J. Lee ${ }^{a}$, P.S. Goh ${ }^{\text {a*, W.J. Lau }}{ }^{\text {a }}$, C.S. Ong a ${ }^{\text {, A.F. Ismail }}{ }^{a}$ \\ ${ }^{a}$ Advanced Membrane Technology Research Centre (AMTEC), Faculty of Chemical and \\ Energy Engineering, Universiti Teknologi Malaysia, 81310 Skudai, Johor, Malaysia \\ *Corresponding Author: peisean@ @etroleum.utm.my
}

\begin{abstract}
The raising oil consumption in oil and gas industries has further exacerbated the disposal of oil waste into the water stream which calls for urging treatment to prevent threats to the human and environment. With some great advantages such as lower membrane fouling rate, lower energy requirement and higher water recovery rate compared to the conventional pressure-driven membrane processes, forward osmosis (FO) has been recognized as a potential candidate for oily wastewater treatment. In this study, a poly[3-(N-2-methacryloylxyethylN,N-dimethyl)-ammonatopropanesulfonate] (PMAPS) incorporated thin film composite (TFC) membrane with excellent anti-fouling properties was fabricated for oily wastewater through forward osmosis process. PMAPS was blended with polyethersulfone (PES) dope solution and cast into PES support layer. Interfacial polymerization (IP) technique was applied to form a thin polyamide (PA) layer atop of the PES support layer. The PMAPS incorporated TFC membranes were characterized for their morphology and surface hydrophilicity. The resultant $1 \%$ PMAPS-TFC membrane exhibited high water flux of $15.79 \pm 0.3 \mathrm{~L} / \mathrm{m}^{2} . \mathrm{h}$ and oil flux of $12.54 \pm 0.8 \mathrm{~L} / \mathrm{m}^{2}$.h when tested in FO mode for oil removal from oily wastewater using 1000 ppm emulsified oily solution as feed solution and $2 \mathrm{M} \mathrm{NaCl}$ as draw solution. The oil rejection up to $99 \%$ was also obtained. Most significantly, PMAPS incorporated TFC membrane outperformed neat TFC membrane with lower fouling propensity for oily waste treatment. When treating $10000 \mathrm{ppm}$ oil emulsion, PMAPS-TFC was able to achieve average flux recovery rate of $97 \%$ while neat TFC only able to achieve $70.8 \%$ of average flux recovery rate.
\end{abstract}


Keywords: Forward osmosis; thin film composite membrane; oily wastewater; zwitterion

\subsection{Introduction}

Oily wastewater is one of the primary pollutants to the environment. The raising oil consumption in oil and gas industries has further exacerbated the disposal of oil waste into the water stream without further treatment [1]. Generally, the wastewater comprises of suspended solids, dispersed oils and dissolved solutes which are harmful to the environment and the water sources. There are few means where wastewater can endanger human such as affecting quality and purity of drinking water and other aquatic sources, threatening human health and aquatic lives, causing meteorological pollution, decreasing crop production as well as devastating natural landscape [2]. Therefore, finding promising approaches for oily wastewater treatment has become a crucial task for the water community.

Membrane technology is a viable approach for oily wastewater treatment as it is useful in completely removing suspended solids and biological degradable organic components from the oily wastewater. A few types of membrane technologies, such as ultrafiltration (UF), nanofiltration (NF) and reverse osmosis (RO), are readily available for oily wastewater treatment. Provided that the feed has too high oil concentration, UF is unable to reduce it to a disposable level. Hereby, a secondary treatment using NF and RO is required to completely remove the remaining oil $[3,4]$. High pressure and high energy are required to work on all these processes. Meanwhile, frequent membrane cleaning as well as larger membrane area are also needed to ensure continuous production[5, 6]. Lately, by applying same principle as other membrane technologies but less energy required, forward osmosis (FO) has won itself an important place in wastewater treatment. The osmotic pressure gradient is utilized as the driving force in FO to separate pure water from feed solution. In addition, FO is also known for its other advantages such as lesser energy required, lower membrane fouling frequency, easier fouling removal [7] and higher water recovery rate [8] compared to pressure-driven membrane processes[9]. Hence FO has been acknowledged as a potential candidate for oil removal[10]. Relatively, membrane fouling rate is lower compared to other pressure driven process, yet its effect is still not negligible hence has to be treated carefully to ensure the durability of membrane [11]. 
Lv et al. [12] has proposed his study on this case using cellulose triacetate (CTA) FO membrane. CTA is chosen in his study as to target good antifouling propensity and cleaning protocol. Apart from that, CTA is selected instead of other type of membranes because it has high resistance towards chlorine and good adsorption of oil droplets and minerals. Compared to the normal cellulose acetate (CA), it is also insensitive to biological degradation and hydrolysis at alkaline conditions. The feed solution (FS) and draw solution (DS) used in his study were actual oily wastewater from Shengli Oilfield (Dongying, China) and $2 \mathrm{M} \mathrm{NaCl}$ respectively. Active layer facing feed solution (AL-FS) orientation was employed in order to obtain better permeate flux and fouling control of the FO membrane as less foulants would be trapped into the porous substrate layer $[5,13]$. However, there is still fouling occurring on the surface of CTA membrane. Significant membrane fouling occurred within 6 hours of operating periods. There is also no obvious pH change in FS after the FO process. The fouled CTA membrane is washed at a high crossflow rate of $33 \mathrm{~cm} \mathrm{~s}^{-1}$ using DI water on both FS and DS sides, which is around four times higher than a normal FO experiment. The flux recovery rate was only around 75-80\%. A study from Han et al. [14] used hollow fiber FO membrane with hydrophilic acetate butyrate $(\mathrm{CAB})$ as the membrane material for substrate layer with outer surface coated by polydopamine (PDA) for oily wastewater treatment. CAB was selected as the membrane material due to its high free volume and better chemical stability than conventional CA caused by bulky functional group [15]. The coated PDA layer onto thin film composite (TFC) layer aimed to modify the pore size of outer surface as well as the hydrophilicity of the $\mathrm{CAB}$ substrate to achieve high permeate flux and low fouling propensity. The FS and DS used in the study were $2000 \mathrm{ppm}$ of oil/water emulsion and $5 \mathrm{~L}$ of $1 \mathrm{M} \mathrm{NaCl}$ solution respectively. The result showed that the modified PDA-TFC-CAB hollow fiber membrane was able to achieve a higher water flux than TFC-CAB hollow fiber membrane due to the improved surface hydrophilicity and altered structure. Apart from that, the PDA-TFCCAB membrane also exhibited excellent antifouling behaviour. The permeate flux only declined slightly 12 hours after running the experiment continuously. Zhang et al. [10] used CTA hollow fiber support and polyvinylidene fluoride (PVDF) hollow fiber membrane in his study for oily wastewater treatment. He used a modified separation system consists of FO and membrane distillation (MD) in his study. The FS used was a synthesized oily wastewater, while the DS used was $2 \mathrm{M}$ of $\mathrm{NaCl}$ solution. Slight increment of fouling was being observed in the beginning of the experiment with increasing oil concentration, and slowly became stable over 24 hours. After 24 hours, no further fouling was observed throughout the operation. A high water flux and oil removal ratio were obtained from the FO process using the modified hollow 
fiber membrane. The hybrid FO-MD system was able to achieve a water recovery of $90 \%$ even in the presence of high feed salinity as well as a nearly complete rejection of oil and $\mathrm{NaCl}$.

In order to improve the separation efficiency, the development of high performance thin film composite (TFC) is highly desired. The introduction of nanomaterials at the substrate and/or the polyamide (PA) layer is deemed one of the most prevailing ways to heighten the performance of TFC. Some common nanomaterials are metal or metal oxide, zeolite, silica, carbon nanotubes (CNTs), graphene oxide (GO)[16]. The incorporated nanomaterials has significantly improved the membrane's physico-chemical properties and separation performance. Nonetheless, further precaution has to be taken because PA crosslink network could be severely disrupted by incompatible nanomaterials. Undesired outcomes can be induced, for instance preventing the polymer end groups to react with other monomers, creating instability and more severe defects [17]. Besides that, some research also showed that the incorporation of zwitterions into membrane active layer brings positive impacts. In brief, a compound which possesses both positive and negative charged groups in the same monomer group is called zwitterion. The opposite charges induce a strong dipole moments which create good interaction between substrate and PA layer. According to Chan et al. [18], the zwitterionfunctionalized single-walled CNTs (Z-SWNT) has improved salt rejection and water permeation flux significantly. Moreover, it also helped to lower the surface fouling in the TFC membrane. The adherence of oil droplets on the membrane surface can be prevented due to the oleophobicity of zwitterions, hence the oily removal and membrane cleaning process can be performed more easily. Although the application of zwitterion could be a tweak to the current membrane technology based on its hydrophilicity, oleophobicity and oil detachment behaviour, the application of Poly[3-(N-2-methacryloxyethyl-N,N-dimethyl)-ammonatopropanesulfonate (PMAPS) in FO for oily wastewater treatment has been scarcely reported. PMAPS is a zwitterion known to exhibit excellent oil detachment behaviour in both water and aqueous $\mathrm{NaCl}$ solution [19]. Our previous study has evidenced the feasibility of PMAPS to act as surface modifier to enhance the performance of the double-skinned TFC membrane for oily wastewater treatment [20]. The objective of this study is to explore the preparation of zwitterion incorporated TFC FO membrane through facile substrate modification. This is the first attempt of fabricating single-skinned zwitterionic TFC FO membranes for oily wastewater treatment. The performance of zwitterion incorporated TFC membrane for oily wastewater treatment was evaluated based on the oil rejection rate, water flux and anti-fouling properties. 


\subsection{Experimental}

\subsection{Materials}

Polyethersulfone (PES) in pellet form (Arkema), Polyvinylpyrrolidone40 (PVP40, Sigma Aldrich) and N-methyl-2-pyrrolidone (NMP, purity > 99.5\%, RC1-Labscan) as addictive were used for fabrication of membrane substrate. m-Phenylenediamine (MPD) and trimesoyl chloride (TMC) from Sigma Aldrich were used to form PA atop PES substrate. To synthesize zwitterionic properties polymer, 2-(dimethylamino)ethyl methacrylate (DMAEMA, 95\%), 2-bromoisobutyrate bromide, diisobutylaluminium hydride (1.0 M in toluene), ethyl 2bromoisobutyrate (EBiB, 98\%), 1,3-propanesultone (99\%), dipyridyl (Bpy, AR), 2bromoisobutyryl bromide (98\%), copper(I) bromide (CuBr, 99\%) and 1-hexyl-3methylimidazolium chloride $(\mathrm{HMImCl})$ were purchased from Sigma Aldrich. Diethyl ether was purchased from RCl-Labscan. Sodium chloride ( $\mathrm{NaCl}$, Merck) was used as draw solution. sodium dodecyl sulfate (SDS, Sigma Aldrich) was used as emulsifying agent for the mixing of crude oil and deionized (DI) water .

\subsection{Synthesis of PMAPS}

The zwitterionic polymer, PMAPS was synthesized as follows [1]. $3.774 \mathrm{~g}$ of N,Ndimethylaminoethyl methacrylate (DMAEMA) was added into $13 \mathrm{~mL}$ of acetone in a $250 \mathrm{~mL}$ flask. The $250 \mathrm{~mL}$ flask was then put on a magnetic stirrer to stir until a homogeneous mixture is formed. Next, $2.442 \mathrm{~g}$ of 1,3-propanesulfonate (1,3-PS) was added drop by drop gradually to the homogeneous mixture formed at ambient temperature under the presence of $\mathrm{N}_{2}$ atmosphere. The reaction was allowed to take place at ambient temperature for 4 hours. After 4 hours, a crude white powder was obtained as the product. Ethanol was further used to filter the product formed. Lastly, it was precipitated in diethyl ether to remove any undesired products. The chemical structure of PMAPS is illustrated in Fig. 1. 


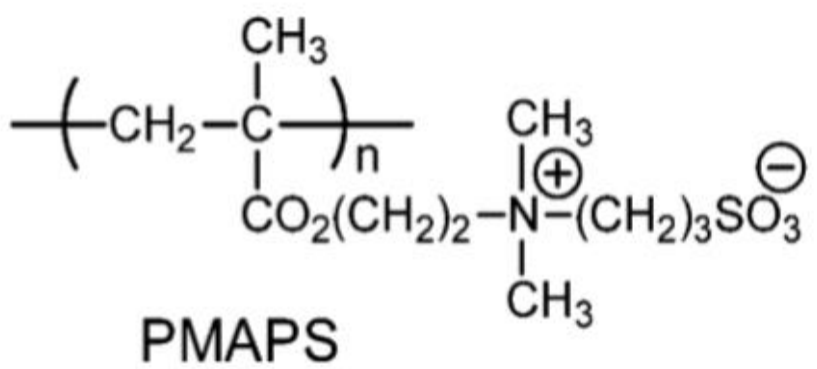

Figure 1: Chemical structure of PMAPS [19, 20]

\subsection{Membrane Preparation}

PES was the polymer used due to its hydrophilicity and good mechanical strength, Nmethyl-2-pyrrolidone (NMP) was the organic solvent used, while polyvinylpyrrolidone (PVP) was used as the membrane pore former. A composition of $18 \mathrm{wt} . \%$ PES, $1 \mathrm{wt} . \%$ PVP, $81 \mathrm{wt} . \%$ NMP, and PMAPS (1\% and 5\%) were physically stirred until homogeneous to prepare polymer dope solution for membrane substrate fabrication. Glass plate, rubber roller, rubber frame, and four clips are used to prepare the PA layers of TFC membranes. Substrate layer was placed on the glass plate while excess water was removed by rubber roller to prevent it from interfering the formation of PA layer because inorganic solvent does not mix well with water. To fix substrate layer on glass plate, rubber frame and clips were used. The fabrication process of PA layer atop of substrate is as follow, the aqueous phase used was $2.0 \mathrm{~g} / 100 \mathrm{ml}$ of mphenylenediamine(MPD)/ $\mathrm{H}_{2} \mathrm{O}$ while organic phase used was $0.1 \mathrm{~g} / 100 \mathrm{ml}$ of trimesoyl chloride(TMC)/cyclohexane. $40 \mathrm{ml}$ MPD aqueous solution was poured on the surface of substrate layer and allowed untouched for $10 \mathrm{~min}$, then removed and cleaned. Also, $40 \mathrm{ml}$ TMC organic solution was poured on the surface of substrate layer and allowed for 1 min reaction before the solution was discard. The resultant membrane was post-treated in oven at $60^{\circ}$ for 8 min. Finally, the membrane was left at room temperature for 30 min before kept in a RO water container until further use.

\subsection{Membrane Characterization}

For characterization of the surface and cross-sectional morphologies of PMAPS-PES support layer and PMAPS-TFC, FESEM (Supra 35VP, Carl Zeiss) was used. Morphological studies are important because it provides information about the structure of membranes that 
can be used to interpret membrane performance and discover possible defects formation. FESEM was also used to measure the thickness of TFC skin layer. Prior to the gold coating, the membrane samples were fractured in liquid nitrogen. To obtain the surface morphologies, scanning was done at a magnification of $\times 5 \mathrm{k}$, which operates at $2 \mathrm{kV}$. The cross-sectional morphologies was determined at magnification of $\times 600$ and/or $\times 20 \mathrm{k}$ which operates at $1 \mathrm{kV}$. Nanowizard 3 from JPK Instruments (Contact mode) was used in order to determine the roughness of PMAPS-PES substrate. Samples were cut into $1 \mathrm{~cm} \times 1 \mathrm{~cm}$ pieces and being attached onto a flat glass slide for AFM scanning using contact mode. $10 \mu \mathrm{m} \times 10 \mu \mathrm{m}$ was the scanning area used. FTIR (Frontier-GPOB, Perkin Elmer) was used to study the functional groups and chemical bonds of PMAPS and PA layer. Analysis was done on the skin layers of the membrane substrate with scanning range of $600 \mathrm{~cm}^{-1}$ to $4000 \mathrm{~cm}^{-1}$. Contact angle system OCA (708381-T, LMS Scientific) was used to carry out water contact angle measurement. The hydrophilicity of the membrane surface can then be further determined from the contact angle measurement and movement of water across membrane. A small rectangular piece of sample of $3 \mathrm{~mm} \times 70 \mathrm{~mm}$ length and width was cut off from the TFC membrane and fixed on the platform. The average reading from10 measurements was reported.

\subsection{Performance Evaluation}

\subsubsection{Pure Water Permeability, Salt Permeability, and Salt Rejection Tests}

A commercial stirred dead-end permeation cell (HP4750, Sterlitech Corp.) was used to conduct the filtration tests. The membrane was placed in the cell with active layer facing inwards. The cell was filled with at least $250 \mathrm{~mL}$ of DI water and membranes were compacted at 16 bar for $30 \mathrm{~min}$ to achieve a steady water flux for testing. Next, $2 \mathrm{~mL}$ of water was collected at 15 bar and the time taken was recorded. To obtain a more accurate result, the test was repeated thrice and the average reading was reported. The pure water permeability coefficient, denoted as $X\left(\mathrm{Lm}^{-2} \mathrm{~h}^{-1} \mathrm{bar}^{-1}\right)$, which is normally abbreviated as LMHB was calculated using Eq. (1)

$X=\frac{V \times 60}{A \Delta t \Delta P \times 1000}$ 
where $V$ is the volume of permeate $(\mathrm{mL}), A$ is the effective area of membrane $\left(0.00146 \mathrm{~m}^{2}\right)$, $\Delta t$ is the operating duration (hr), $\Delta P$ is the applied pressure difference (bar).

For salt flux and salt rejection test, DI water was used to compress the membrane for 30 min before carrying out the experiment. After that, the process was similar but only the DI water was replaced by $2000 \mathrm{ppm}$ of $\mathrm{NaCl}$ solution. It was allowed to run for another $15 \mathrm{~min}$ to achieve a stabilized flux. $2 \mathrm{~mL}$ of salt permeate was collected and the time taken was recorded. The process was repeated thrice to get an average reading. The salt rejection was determined based on the difference between feed and permeate conductivity using a conductivity meter (HC3010, Trans Instruments). The salt permeability coefficient, $Y\left(\mathrm{Lm}^{-2} \mathrm{~h}^{-1} \mathrm{bar}^{-1}\right)$ and salt rejection, $R_{\mathrm{S}}(\%)$ were calculated using Eq. (2) and (3) respectively.

$Y=\frac{V \times 60}{A \Delta t \Delta P \times 1000}$

$R_{\mathrm{s}}=\left(1-\frac{C_{p}}{C_{f}}\right) \times 100 \%$

where $C_{\mathrm{p}}$ and $C_{\mathrm{f}}$ represent the conductivity of the permeate and the feed $(\mathrm{S})$ respectively.

\subsubsection{Water Flux, Oil Rejection, and Anti-fouling Performance Tests}

The steps in the previous tests were repeated for anti-fouling studies. DI water and salt solution used were replaced with 1,000 ppm of crude oil (RE110, Malaysia) in water. The crude oil was mixed with SDS in a ratio of 1:9 in order to obtain a homogenous oil emulsion which was similar to steps used by [14]. Oil rejection can be measured using several means such as UV-vis spectrometer or TOC analyser. Since using TOC analyser is more time-consuming, to obtain the oil rejection, the oil feed and permeate concentrations were determined by using UV-vis spectrometer (DR2800, Hach). The absorbance was being measured at $273 \mathrm{~nm}$ which showed the peak where most absorbance occurred. The water flux, $J_{\mathrm{v}}\left(\mathrm{Lm}^{-2} \mathrm{~h}^{-1}\right)$, also known as LMH and oil rejection, $R_{\circ}(\%)$ were calculated according to Eq. (4) and (5)

$J_{\mathrm{V}}=\frac{V \times 60}{A \Delta t \times 1000}$ 
$R_{\mathrm{o}}=\left(1-\frac{C_{p}}{C_{f}}\right) \times 100 \%$

Where $C_{\mathrm{p}}$ and $C_{\mathrm{f}}$ represents concentration of oil permeate and oil feed (abs) respectively.

After the oil filtration test, the membrane was removed from the system and allowed to wash with DI water for $20 \mathrm{~min}$. Next, anti-fouling test was conducted over a total period of 960 min in RO mode, which consisted of four cycles, in order to observe the flux change along the test. After each cycle, the membrane was washed with DI water for $20 \mathrm{~min}$. Besides, $5000 \mathrm{ppm}$ and $10000 \mathrm{ppm}$ emulsified oil solution were also used to evaluate the anti-fouling performance of the membrane in highly concentrated oily waste water.

\subsubsection{Forward Osmosis Test}

The effective membrane area of the cross-flow FO cell for membrane to attach on is $20.02 \mathrm{~cm}^{2} .1$ bar was the system operating pressure throughout the process for FS and DS circulation with the help of two variable speed pumps. AL-FS orientation was used, while the velocity of FS and DS were fixed at $32.72 \mathrm{~cm} / \mathrm{s}$. The process was operated at ambient temperature. The feed solution used was $1,000 \mathrm{ppm}$ of oily wastewater while the draw solution used was 2.0 M of concentrated salt solution. In order to obtain a precise water flux, a digital weight balance was placed at the bottom of feed solution tank. $500 \mathrm{ml} / \mathrm{min}$ was the cross flow rate used to circulate both FS and DS. Fig. 2 illustrates the setup for the FO system constructed for this study. 


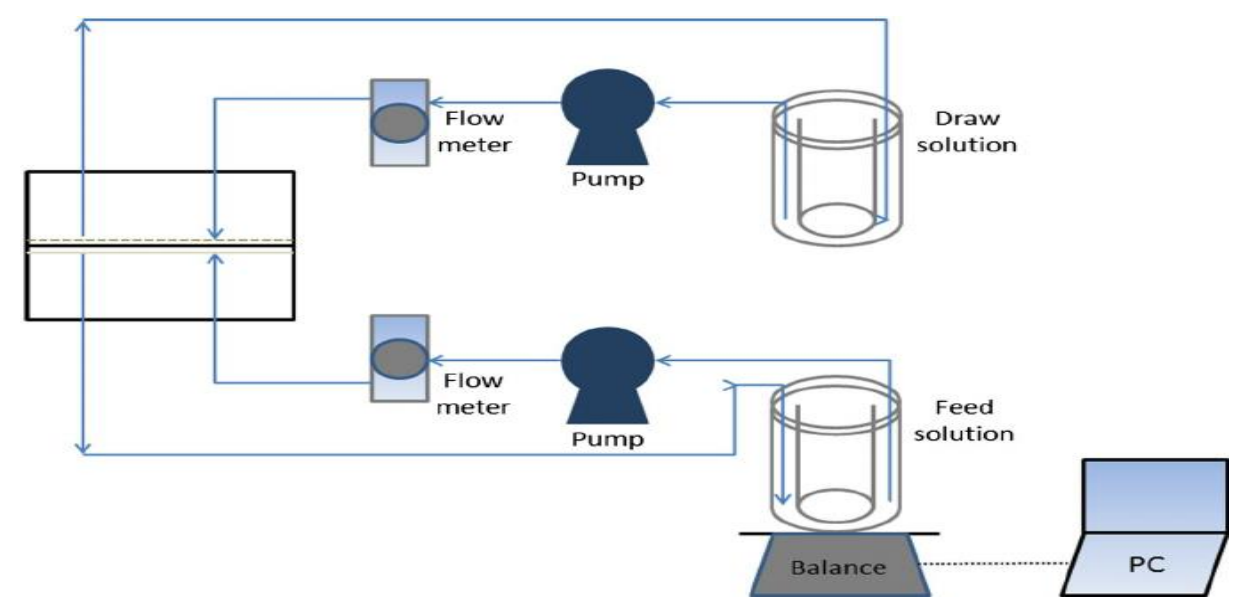

Figure 2: Schematic diagram of a bench-scale forward osmosis setup for testing of the TFC membrane setup [21]

The water flux, $J_{\mathrm{v}}\left(\mathrm{Lm}^{-2} \mathrm{~h}^{-1}\right)$ was calculated by applying Eq. (6):

$J_{\mathrm{V}}=\frac{\Delta V}{A \Delta t \times 1000}$

where $\Delta V$ is the volume change of the feed solution $(\mathrm{mL}), A$ is the effective area of membrane $\left(0.002002 \mathrm{~m}^{2}\right) \Delta t$ is the operating time interval (hr).

Meanwhile, the reverse salt flux, $\left(\mathrm{gm}^{-2} \mathrm{~h}^{-1}\right)$ also known as $\mathrm{gMH}$ was obtained by applying Eq.

(7) based on change in volume of feed solution and salt concentration:

$J_{\mathrm{s}}=\frac{\Delta C_{f} \Delta V}{A \Delta t \times 1000}$

where $\Delta C_{\mathrm{f}}(\mathrm{g} / \mathrm{L})$ and $\Delta V(\mathrm{~mL})$ are the change in salt concentration in feed solution and volume of feed solution measured at the beginning and the end of time interval accordingly. The conductivity of the feed solution was measured by using a conductivity meter, and the respective values were converted into concentration using a calibration graph.

Besides, the oil flux, $J_{\mathrm{o}, \mathrm{FO}}(\mathrm{gMH})$ can be calculated using Eq. (8)

$J_{\mathrm{o}, \mathrm{FO}}=\frac{\Delta C_{d} \Delta V}{A \Delta t \times 1000}$

where $\Delta C_{\mathrm{d}}(\mathrm{g} / \mathrm{L})$ and $\Delta V(\mathrm{~mL})$ are the change in oil concentration and volume of draw solution measured at the beginning and the end of time interval accordingly. UV-vis spectrometer (DR 
2800, Hach) was used to obtain the oil concentration of draw solution by converting the absorbance of the draw solution into oil concentration. Fig. 3 illustrates concentration profile of TFC membrane.

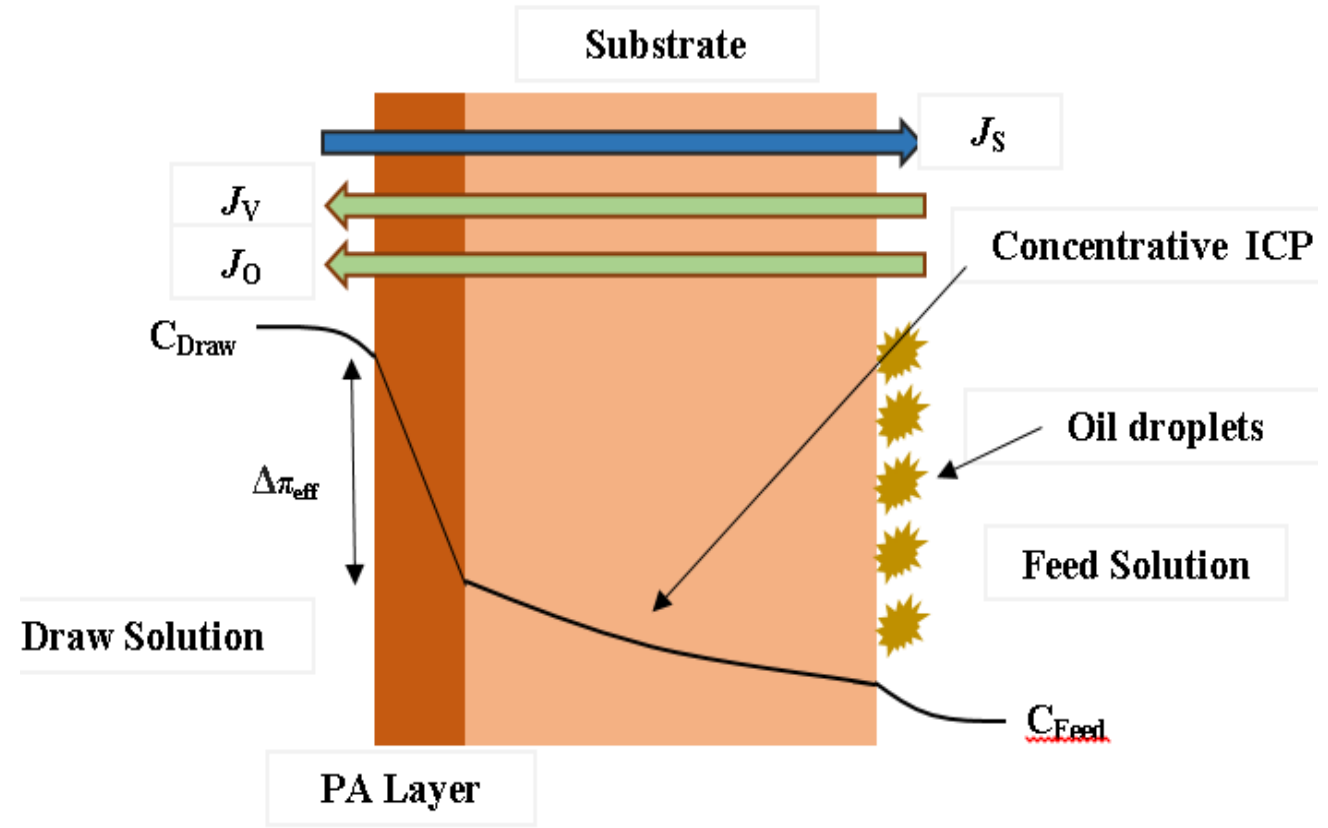

Figure 3: Concentration profile of TFC Membrane

\subsection{Results and Discussion}

\subsection{Membrane Characterization}

\subsubsection{FTIR Characterization}

From FTIR characterization results, the successful incorporation of PMAPS could be confirmed by observing the characteristic bands as shown in the spectra in Fig. 4. At around $1720 \mathrm{~cm}^{-1}$, the characteristic band of $-\mathrm{O}-\mathrm{C}=\mathrm{C}$ group which belongs to the PMAPS polymer could be found [22], However on neat PES substrate and neat TFC membrane, such characteristic band was clearly absent. Such characteristic band appeared on both PMAPS-PES substrate and PMAPS-TFC membranes that validates the successful incorporation of PMAPS. Besides, different PMAPS loading did not affect much the intensity of characteristic band formed. In addition, the presence of aromatic ring stretching (amide II) could be noticed at $1607.56 \mathrm{~cm}^{-1}$ in all TFC membranes, which validates the successful formation of PA layer. 


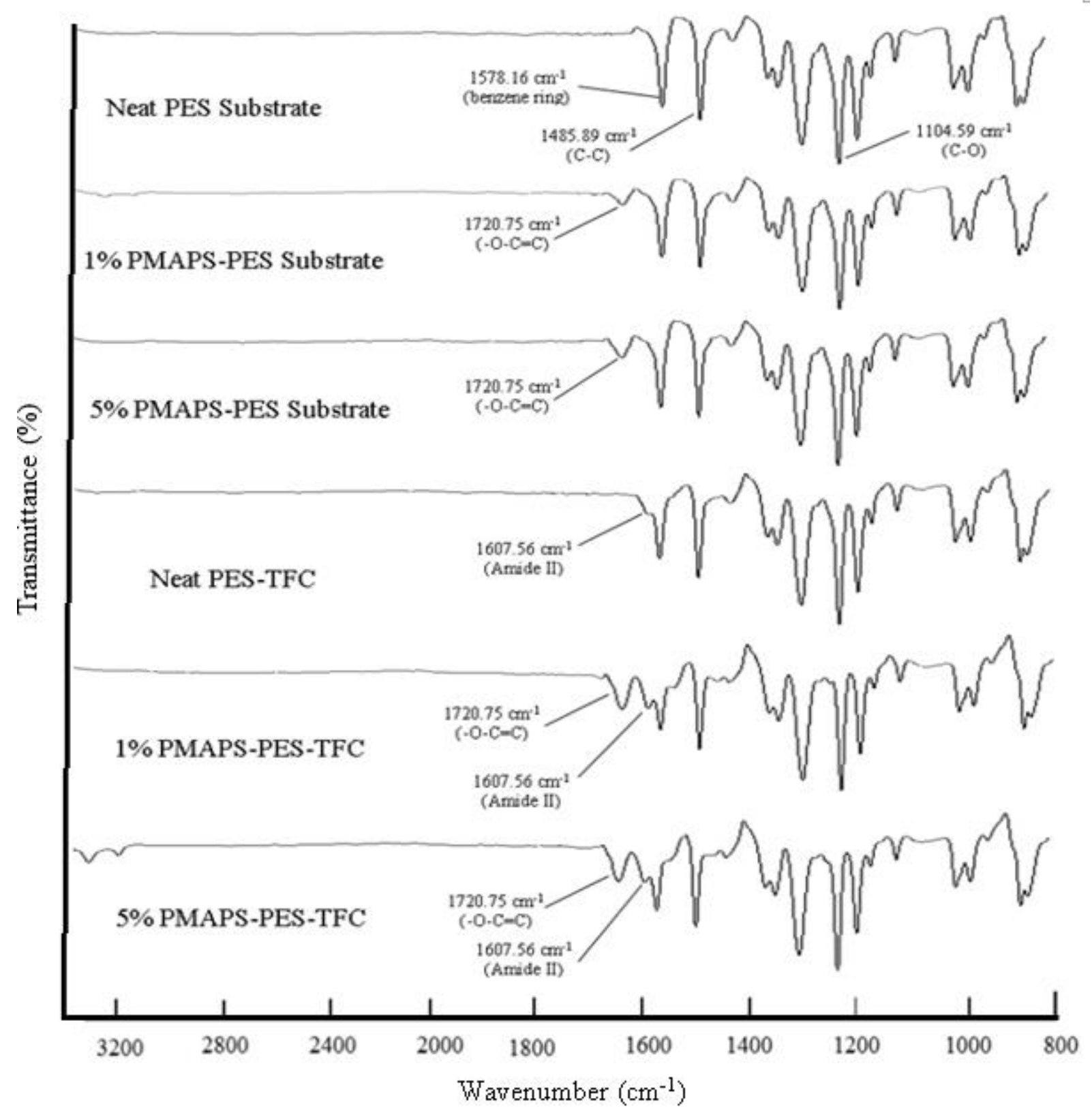

Figure 4: FTIR spectra of PES substrate membranes and TFC membranes

\subsubsection{Membrane Surface Wettability}

Water contact angle measurements were carried out to evaluate the hydrophilicity of membrane surface. The contact angle of $1 \%$ PMAPS-TFC membrane was $35.31 \pm 0.78^{\circ}$ while of the 5\% PMAPS-TFC membrane was $42.32 \pm 12.29^{\circ}$. Higher water contact angle means lower surface hydrophilicity. Comparing to the contact angle of neat TFC membrane $\left(57.65 \pm 0.94^{\circ}\right)$, it shows that PMAPS can improve the membrane surface hydrophilicity.

The underwater oleophobicity was also evaluated by measuring the oil contact angle under water. The hydration layer affects the underwater oleophibicity of the membrane. The 
stronger the hydration layer, the higher the contact angle of oil will be, which also indicates a better membrane oleophobicity. As expected, the neat TFC membrane gave lower underwater oil contact angle of $98.51 \pm 0.89^{\circ}$. On the contrary, $1 \%$ PMAPS-TFC exhibited $156 \pm 0.61^{\circ}$ owing to its excellent hydration property[23]. Meanwhile, 5\% PMAPS-TFC again exhibited unstable result which was $135 \pm 10.46^{\circ}$.

\subsubsection{FESEM \& AFM Characterizations}

Fig. 5 and Fig. 6 represent the cross sectional and surface morphology of PES substrate layer and TFC membrane, accordingly. Fig. 5 shows that all membranes exhibited long fingerlike structure and highly porous middle part. The membranes formed consisted of 3 primary layers, which were: (1) non-woven fabric which acts as a support layer; (2) microporous support membrane formed by phase inversion on top of non-woven fabric layer; (3) ultrathin active layer formed via IP process on the top of entire membrane accounted for salt and oil rejection. Water movement across the membrane was eased by the finger-like structure, and greatly reduced water transport resistance and internal concentration polarization (ICP). With $1 \%$ loading of PMAPS, it could be observed that the finger-like pores distributed uniformly throughout the membrane substrate layer. Nevertheless, high loading of 5\% PMAPS caused the formation of non-uniform pores of different sizes distributed unevenly throughout the membrane structure. Structure and pore distribution of both neat TFC and 1\% PMAPS-TFC membrane were almost similar and were expected to render enhanced water flux. The voids were small when close to the surface and became increasingly huge while getting closer to bottom layer. From Fig. 6, it can be seen the PA layer formed from IP process exhibited typical ridge-valley with finely dispersed grainy structure which can be commonly found in PA layer formed from IP process of MPD/TMC monomers[24]. Upon the formation of PA layer, the membrane surface became rougher. As shown from the AFM images in Fig. 7, the surface roughness increased with $\mathrm{R}_{\mathrm{a}}$ parameter from neat membrane $(38.22 \mathrm{~nm})$, $1 \%$ PMAPS-TFC membrane $(65.97 \mathrm{~nm})$ to $5 \%$ PMAPS-TFC membrane $(99.61 \mathrm{~nm})$. It can be concluded that the surface roughness increases proportionally with the loading of PMAPS. The increase in surface roughness was probably caused by the aggregation of PMAPS of membrane surface. It also further supports the earlier discussion on the uneven membrane pore distribution which was caused by the excessive loading of PMAPS as pore formation can be directly associated to the surface roughness[25]. Apart from that, the pore diameter of support layer for neat TFC, $1 \%$ PMAPS-TFC and 5\% PMAPS-TFC was $0.059 \mu \mathrm{m}, 0.061 \mu \mathrm{m}$ and $0.23 \mu \mathrm{m}$, respectively. It 
was noticed that the average pore diameter of 5\% PMAPS-TFC was around 3 times larger than that of neat TFC and 1\% PMAPS-TFC. All the PA layer formed had thickness of around 0.1 $\mu \mathrm{m}$. It is important to know that the pore size of the support layer in contact with PA layer must be smaller than the thickness of PA layer in order to support the PA layer under high pressure. The interface between the PA layer and substrate should remain intact and close together so that the path length for permeate diffusion is short[26]. In this case, due to the large pore diameter of 5\% PMAPS-TFC, the PA layer was not able to form well atop it.

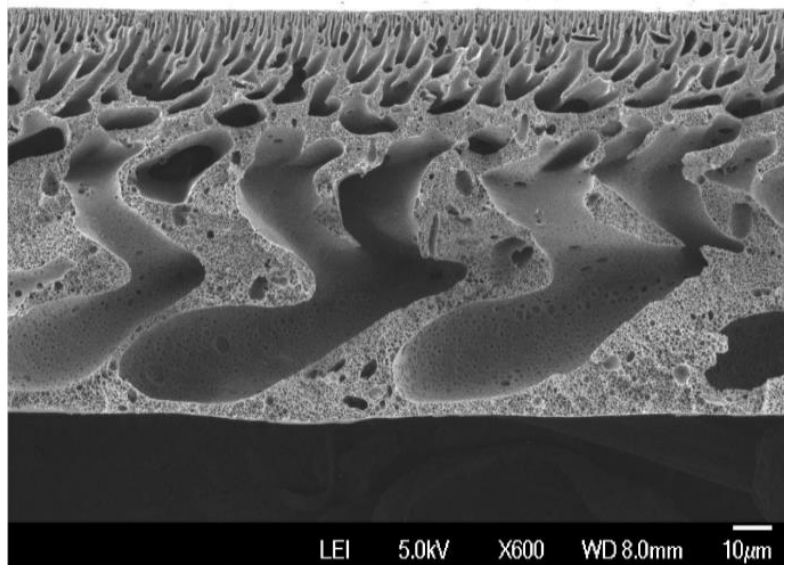

(a)

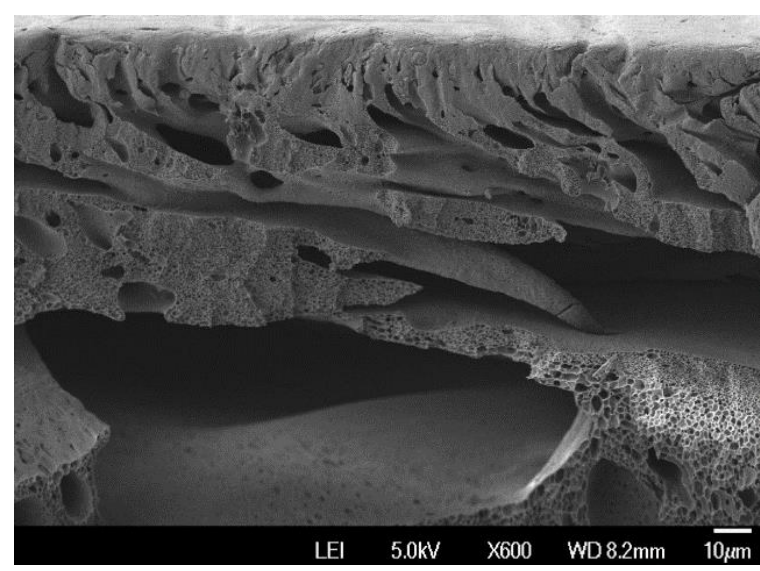

(b)

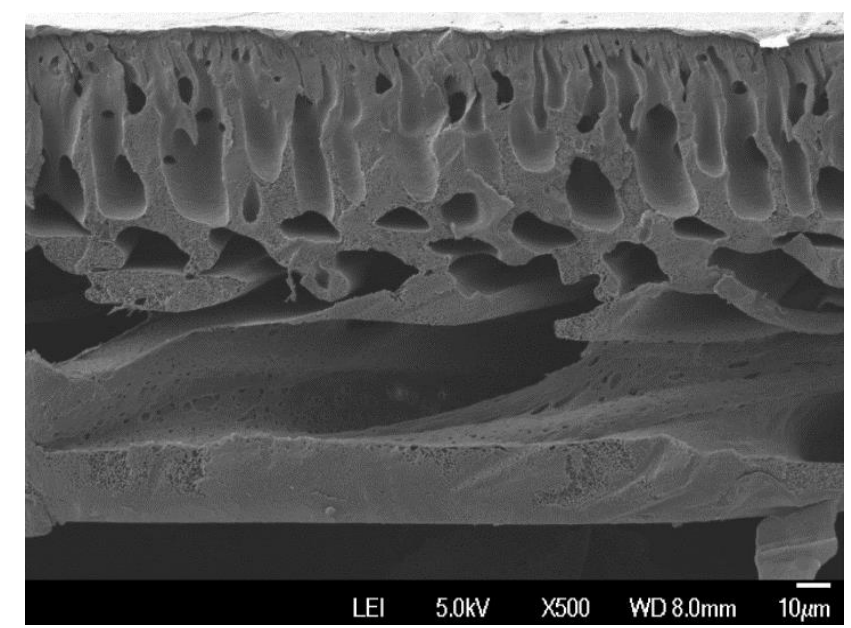

(c)

Figure 5: FESEM images of cross-sectional morphology, (a) 1\% PMAPS-TFC, (b) 5\% PMAPS-TFC (c) neat TFC 


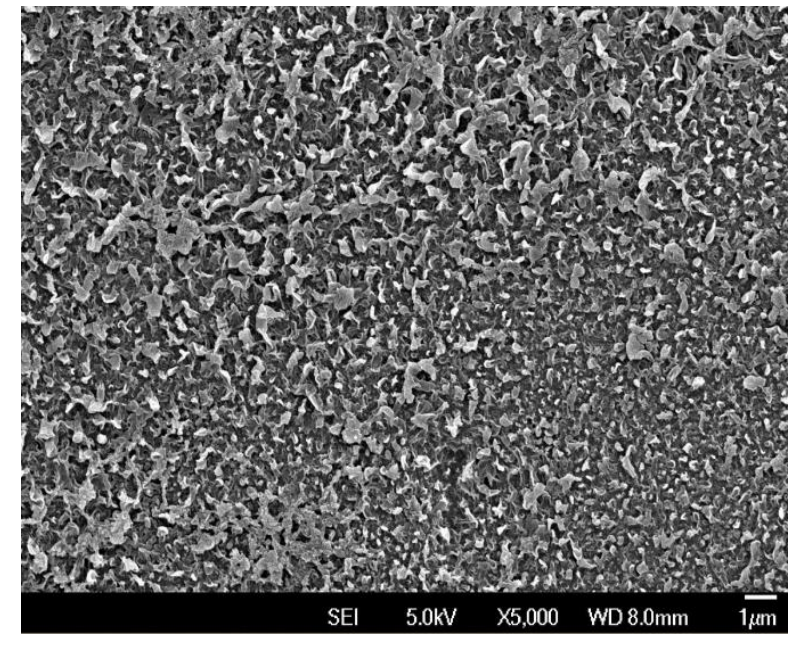

(a)

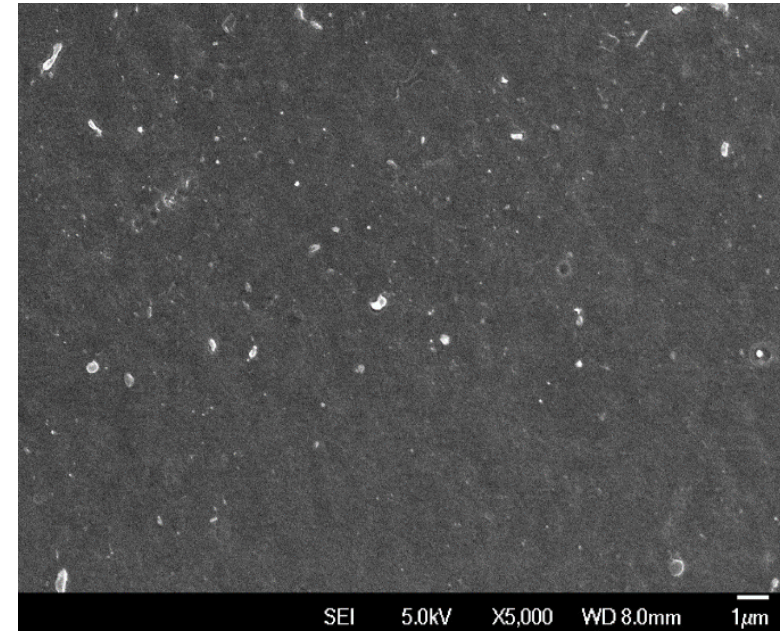

(b)

Figure 6: FESEM images of (a) TFC surface with PA layer, (b) PES substrate surface

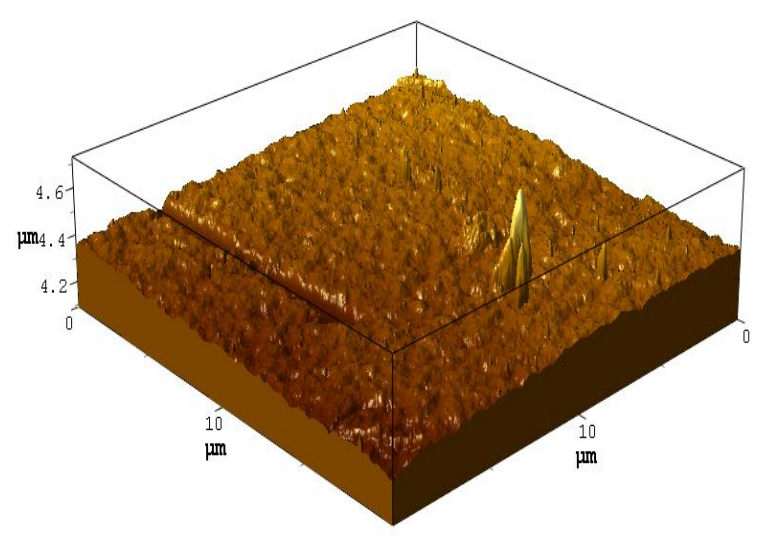

(a)

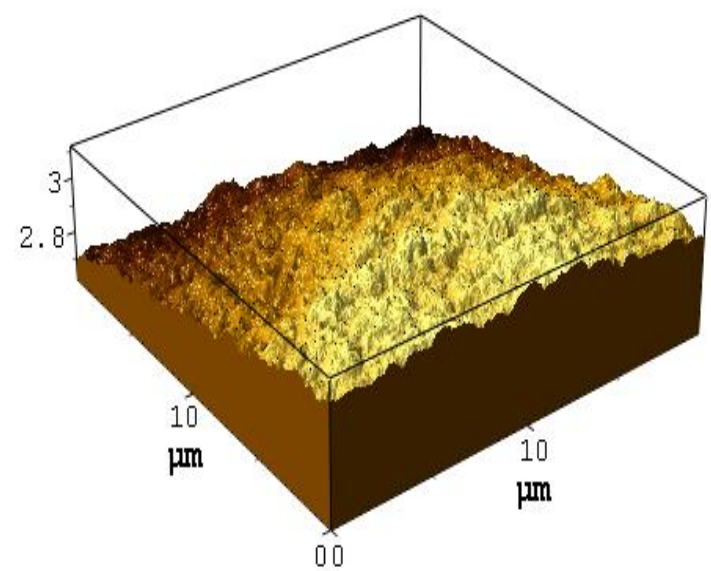

(b)

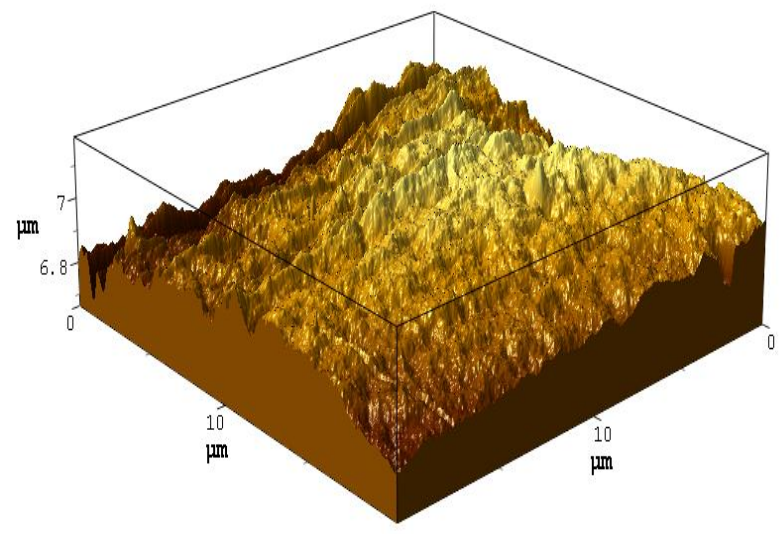

(c)

Figure 7: AFM images of (a) neat TFC (b) 1\% PMAPS-TFC (c) 5\% PMAPS-TFC

\subsection{Oily Wastewater Treatment Performance Testing}


From Fig. 8, it shows the comparison of the solute/solvent permeability and rejection of neat TFC membrane and PMAPS-TFC membrane with $1 \%$ and $5 \%$ of PMAPS loading. Permeability test was conducted in RO mode as FO mode does not require pressure. 1\% PMAPS-TFC membrane granted higher pure water coefficient and salt permeability coefficient compared to neat TFC membrane, which were 0.69 LMHB and 0.56 LMHB respectively for 1\% PMAPS-TFC membrane, and 0.57 LMHB and 0.45 LMHB respectively for neat TFC membrane. Nevertheless, permeability exhibited by 5\% PMAPS-TFC was much higher which was 1.81 LMHB for salt permeability and 1.83 LMHB for pure water permeability. When comparing permeability coefficient with rejection, both neat TFC and 1\% PMAPS-TFC membranes exhibited high salt and oil rejection rates. Neat TFC exhibited $96.2 \%$ of salt rejection and $98.5 \%$ of oil rejection meanwhile 1\% PMAPS-TFC membrane showed $95.8 \%$ of salt rejection and $99.9 \%$ of oil rejection. On the other hand, 5\% PMAPS-TFC membrane had extremely low rejection rate which are $26.5 \%$ and $29.6 \%$ for salt and oil rejections, respectively. It can be further deduced that high loading of PMAPS has adverse impact on the thin film PA layer formation. The poor performance of 5\% PMAPS-TFC was most likely caused by the random non-uniform pores distribution across the membrane surface, and the uneven macroporous distribution upon the addition of 5\% PMAPS had severely affected the MPD and TMC polymerization on the substrate during IP process, thus forming a defective PA selective layer. Also, the relatively big pore size of 5\% PMAPS-TFC support layer to PA layer thickness also caused PA layer not able to form well atop it. In order to prevent the undesirable trade-off effect as happened in 5\% PMAPS-TFC, moderate amount of PMAPS zwitterion should be used during the membrane formation. The higher permeability coefficients of $1 \%$ PMAPS-TFC than neat TFC membrane is attributed to the improved hydrophilicity of PMAPS as proven from the contact angle measurement. 


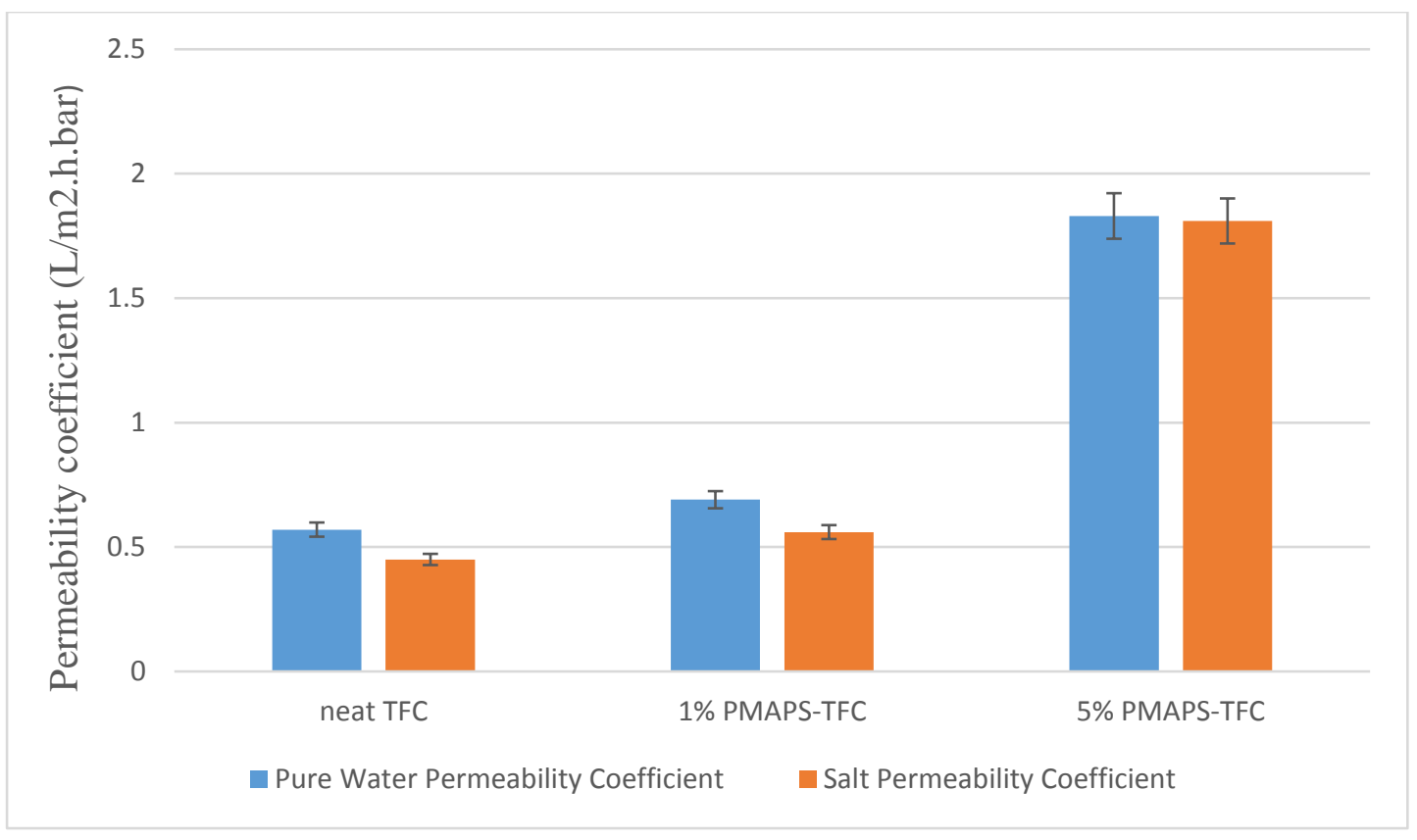

(a)

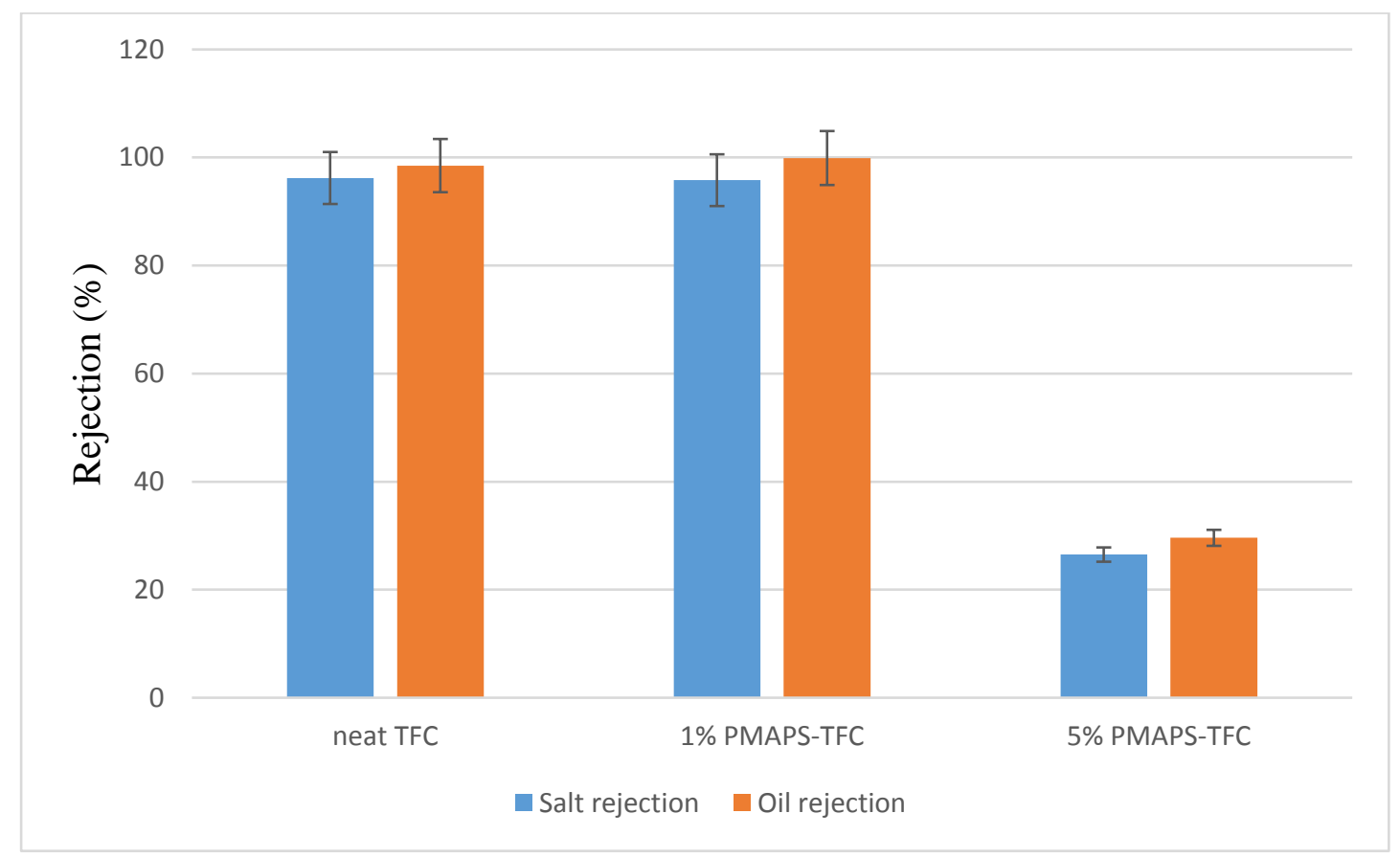

(b)

Figure 8: Transport properties of neat TFC and PMAPS-TFC membranes, (a) pure water permeability coefficient and salt permeability coefficient and (b) salt rejection and oil rejection for DI water, $2000 \mathrm{ppm} \mathrm{NaCl}$ solution and 1,000 ppm emulsified oil solution tested under RO mode at 15 bar. 
Water flux test was conducted in FO mode. Tab. 1 shows comparison between the flux performance of neat TFC membrane and PMAPS-TFC membrane. 1\% PMAPS-TFC membrane exhibited higher water flux and reverse solute flux compared to neat TFC by using $2 \mathrm{M} \mathrm{NaCl}$ as the draw solution and 1,000 ppm of oil emulsion as feed solution in AL-FS mode. In addition, 1\% PMAPS-TFC membrane granted higher water flux, which was $15.79 \mathrm{LMH}$ than neat membrane which was $12.54 \mathrm{LMH}$, while maintaining high rejection rate up to $99.9 \%$. Besides, the ICP of 1\% PMAPS membrane was also lower, which can be implied from its higher oil flux, $J_{\mathrm{o}}$ which was $0.0093 \mathrm{gMH}$ and reverse solute flux, $J_{\mathrm{s}}$ which was $4.21 \mathrm{gMH}$ when compared to neat TFC membrane which were $0.0079 \mathrm{gMH}$ and $3.56 \mathrm{gMH}$ respectively. This is due to high deposition of solutes on neat TFC membrane's porous support layer which led to higher ICP, hence resulted in lower water flux[27]. It also further indicates that $1 \%$ PMAPSTFC membrane possessed greater antifouling properties compared to neat TFC membrane. However, the anti-fouling test would not be carried out on 5\% PMAPS-TFC membrane despite its much higher overall flux than 1\% PMAPS-TFC membrane, due to the extremely low oil rejection rate which is highly undesired.

Table 1: Flux performance of the neat TFC membrane and PMAPS-TFC membrane

\begin{tabular}{llll}
\hline Types of Membranes & $\begin{array}{l}\text { Water Flux, } J_{\mathrm{v}} \\
(\mathrm{LMH})\end{array}$ & $\begin{array}{l}\text { Reverse Solute } \\
\text { Flux, } J_{\mathrm{s}}(\mathrm{gMH})\end{array}$ & $\begin{array}{l}\text { Oil Flux, } J_{\mathrm{o}} \\
(\mathrm{gMH})\end{array}$ \\
\hline neat TFC & 12.54 & 3.56 & 0.0079 \\
\hline 1\% PMAPS-PES & 15.79 & 4.21 & 0.0093 \\
\hline $5 \%$ PMAPS-PES & 31.58 & 24.52 & 0.39 \\
\hline
\end{tabular}

\subsection{Anti-fouling Test}

The antifouling behaviour of PMAPS incorporated TFC was studied through a prolonged filtration process. Fig. 9 (a), 9 (b) and 9 (c) compare the water flux profile with flux recovery over a period of 960 min filtration process consisting of four cycles using $1000 \mathrm{ppm}$, $5000 \mathrm{ppm}$ and $10000 \mathrm{ppm}$ of emulsified crude oil respectively. In this test, only 1\% PMAPSTFC membrane and neat TFC membrane were used for comparison. For 5\% PMAPS-TFC membranes, the entering of oil particles from the emulsified oil feed into the porous support caused internal fouling while the deposition of oil particles onto the membrane surface caused external fouling which in turn resulted in significant flux drop. As a result, the transport resistance was increased. It can be seen that the both $1 \%$ PMAPS-TFC and neat TFC 
membranes suffered from fouling issue. However, regardless of the oil concentration used, it can be noticed that the neat TFC membrane experienced a much more severe flux decline during the separation process. On the contrary, for 1\% PMAPS-TFC, a slower and milder flux declined was noticed, indicating the improved anti-fouling behaviour. Also, comparing the performances of both membranes, it can be noticed that at the beginning of each new cycle after washing the membrane with DI water, PMAPS-TFC was able to recover its water flux close to the original flux while neat TFC membrane exhibited a lower recovery rate. Apart from that, PMAPS-TFC also had lower flux drop at the end of each cycle compared to that of neat TFC membrane. It can be noticed that the water flux decline for both neat TFC and PMAPS-TFC can be divided in two distinctive fouling stages, which were an exponential flux decline followed by a near linear decline of the water flux. With increasing oil concentration, it can be observed that the rate of flux decline from exponential flux decline stage to linear flux decline stage accelerated. With 1000 ppm mild oil emulsion, the flux decline rate of neat TFC and $1 \%$ PMAPS-TFC was nearly comparable. However as the oil emulsion became more concentrated (5000 ppm and $10000 \mathrm{ppm})$, the flux decline rate of the neat TFC became significantly higher than that of TFC, showing that superior antifouling behaviour of PMAPS which is able to handle high oil concentration. For 1000 ppm oil emulsion, the average flux drop is $34.6 \%$ and $40.6 \%$ for PMAPS-TFC and neat TFC, respectively. As the oil concentration increased, PMAPS-TFC was able to maintain its average flux drop at $33.4 \%$ for 5000 ppm oil emulsion and $34.8 \%$ for $10000 \mathrm{ppm}$ oil emulsion. However, the flux drop phenomena with neat TFC became more severe as the oil concentration increased, which were $45.1 \%$ for $5000 \mathrm{ppm}$ oil emulsion and 51.2\% for 10000 ppm oil emulsion. For water flux recovery, PMAPS-TFC was able to achieve more than $97 \%$ recovery rate regardless of the oil concentration. On the other hand, neat TFC membrane was not able to recover to its original flux after each cycle of washing. As shown in Fig. 10, the presence of PMAPS eases the removal process of oil particles on the internal pore wall of membrane. The hydrogen bonding formed between $\mathrm{SO}_{3}$ functional group of PMAPS and $\mathrm{H}_{2} \mathrm{O}$, and a hydration layer formed between water molecules acts as an outer wall to prevent oil particles to adhere on the membrane internal pore surface. Thus, the oil particles attach to hydration layer instead of membrane wall. Hence, it can be feasibly removed by rinsing with water[20]. Apart from hydration layer, zwitterionic support layer also carried salt responsive properties, whereby their configuration changed from coiled state to swelling state in the presence of chloride salt[28]. The ion-stimulated conversion demeanor could induce driving force which discharged the accumulated foulants from membrane surface[29]. 


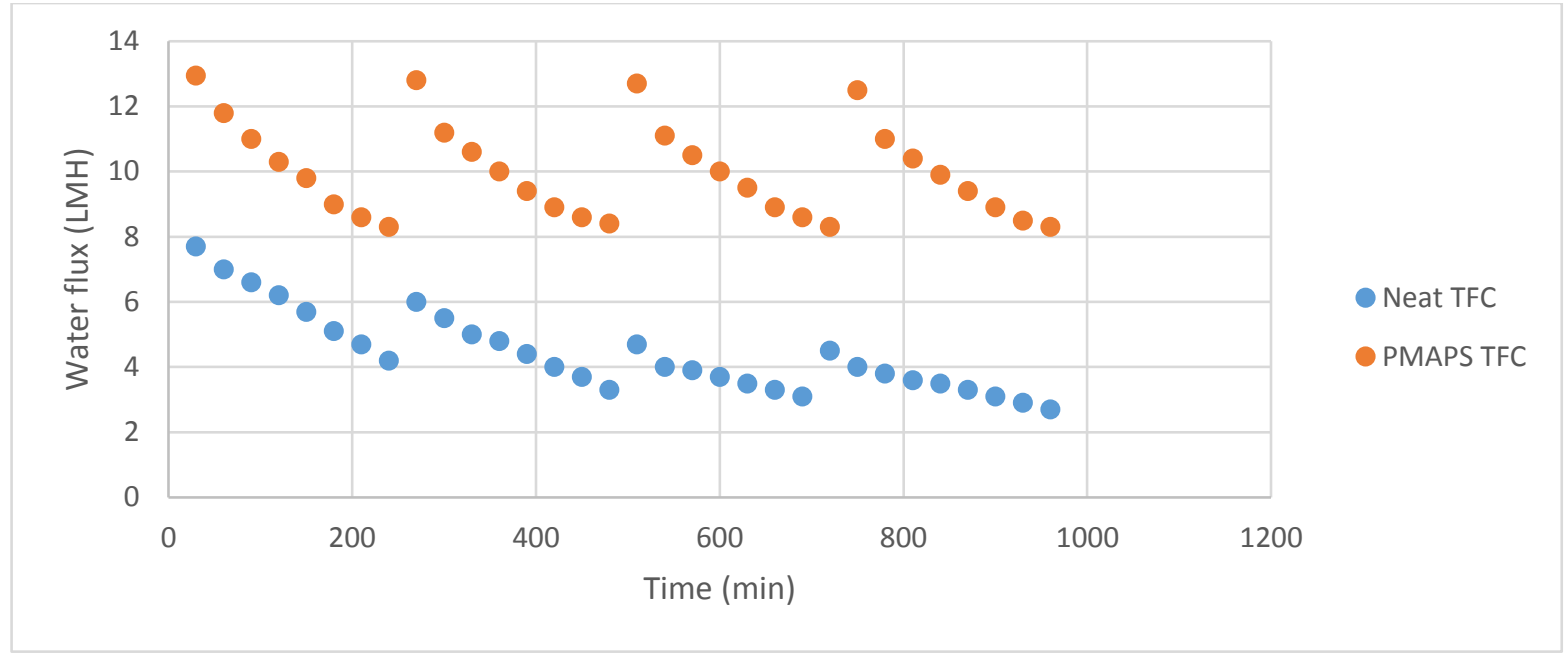

(a)

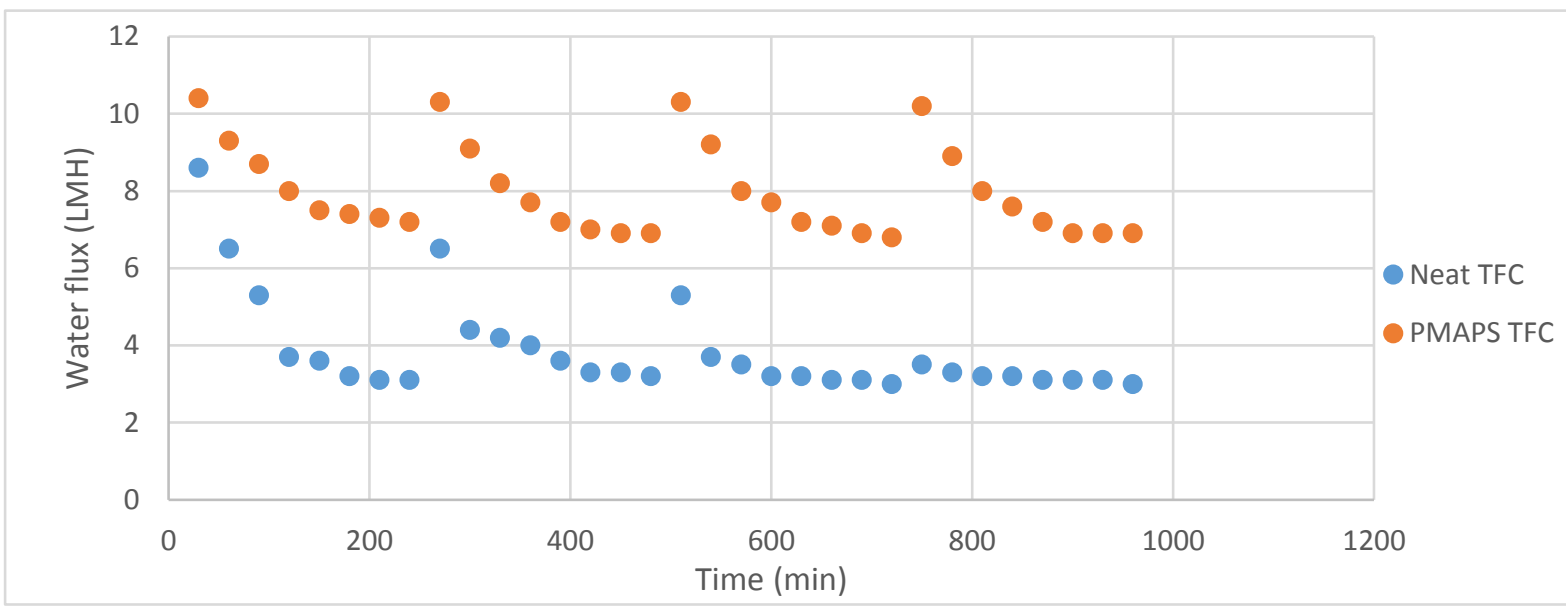

(b)

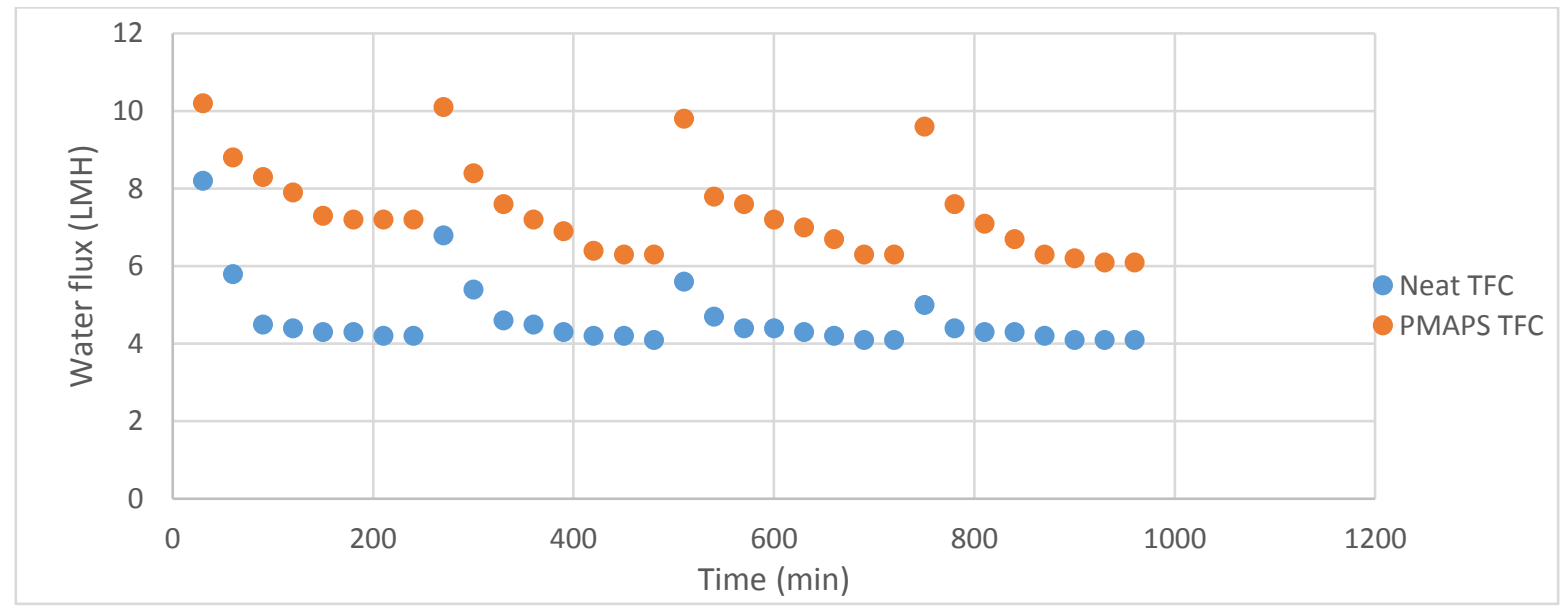

(c)

Figure 9: Comparison of water flux for PMAPS-TFC and neat TFC membranes at (a) 1000 ppm oil emulsion, (b) 5000 ppm oil emulsion, (c) 10000 ppm oil emulsion over 960 minutes of four cycles operation. 


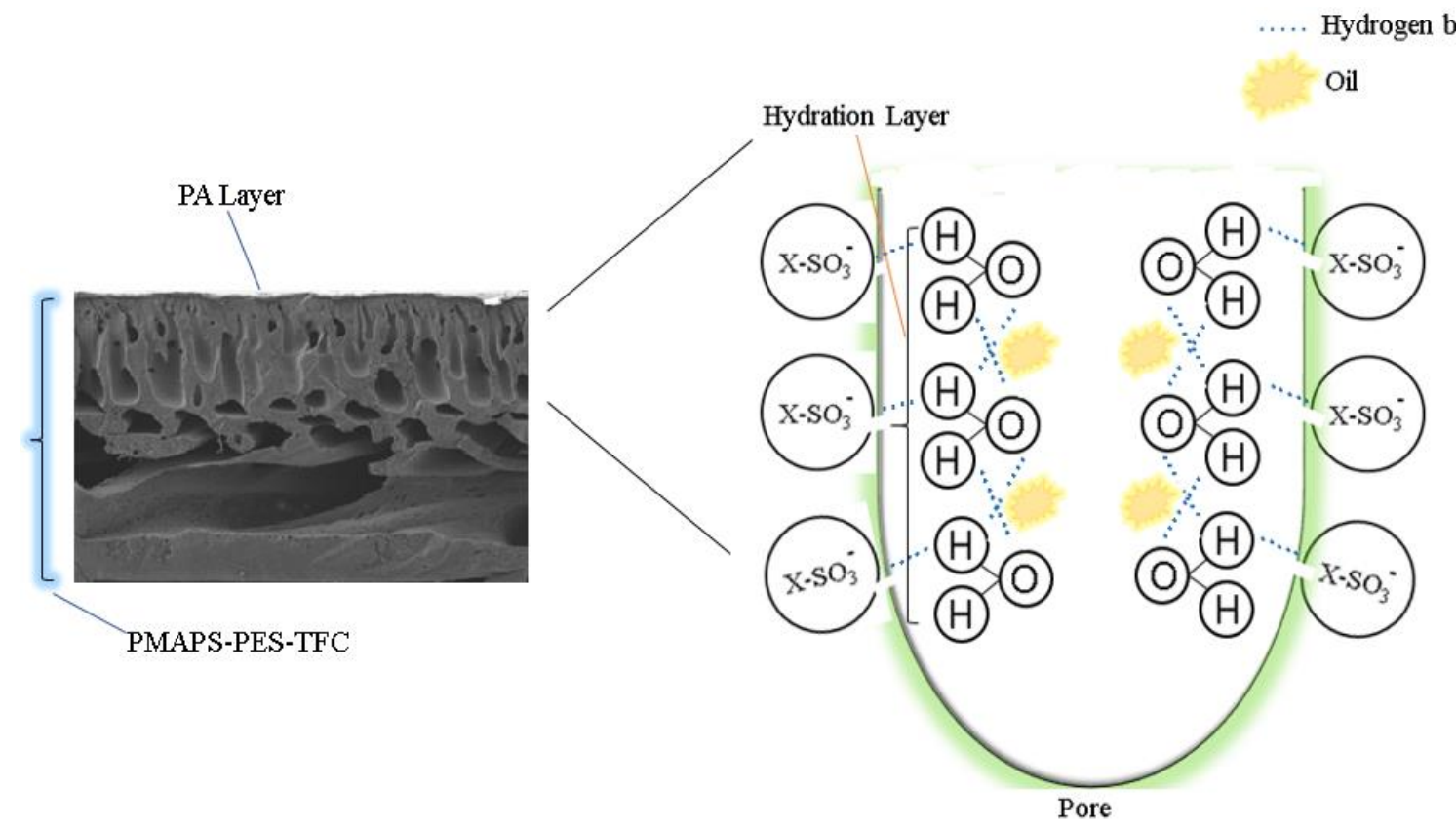

Figure 10: Schematic diagram of hydrogen bonding and electrostatic attraction of PMAPSTFC membrane to prevent oil adhesion on membrane surface

\subsection{Conclusion}

In this study, a PMAPS incorporated TFC membrane has been prepared to treat highly concentrated oil emulsion. The characteristic band of $1715 \mathrm{~cm}^{-1}$ signifies that the PMAPS incorporation has been successful. Due to the great hydrophilicity of PMAPS, $1 \%$ PMAPSTFC exhibits lower water contact angle $\left(35.31 \pm 0.78^{\circ}\right)$ compared to neat membrane $\left(57.65 \pm 0.94^{\circ}\right)$. To ease the water transportation across the membrane, all the membranes are found out to have long-finger like structure and highly porous middle. Besides, the membrane's hydrophilicity get to be enhanced with aid of the hydrophilic PMAPS, which results in higher water flux of 15.79 $\mathrm{LMH}$ than neat membrane of $12.54 \mathrm{LMH}$ using $2 \mathrm{M} \mathrm{NaCl}$ as draw solution. Meanwhile, it also exhibits highly desired oil rejection up to $99.9 \%$, and oil flux of 0.0093 $\mathrm{gMH}$. Under RO mode, the pure water permeability coefficient and salt permeability coefficient of PMAPS-TFC membrane obtained are also excellent which are 0.69 and 0.56 LMHB accordingly. Nevertheless, immoderate PMAPS loading can cause random distribution of non-uniform pores of different sizes throughout the membrane structure resulting in defective membrane structure and characteristic hence poor performance. For $10000 \mathrm{ppm}$ oil emulsion, the water flux drop of both PMAPS and neat TFC membranes are $34.8 \%$ and $51.2 \%$ 
accordingly after $960 \mathrm{~min}$ of filtration cycle. Apart from that, PMAPS-TFC can achieve more than $97 \%$ of flux recovery rate for each oil concentration at the starting point of each new cycle after being rinsed by DI water. At the same time, neat TFC membrane exhibits a relatively low average flux recovery rate which is $70.8 \%$ for $10000 \mathrm{ppm}$ oil emulsion. The great hydrophilic characteristic of PMAPS can be further evidenced from its remarkable anti-fouling properties that helps to prevent internal and external fouling. The present study shows the feasibility of incorporating PMAPS into the TFC substrate layer via a facile blending method. Also, it provides a versatile approach to innovate and fabricate TFC membrane with superior antifouling properties for highly concentrated oily wastewater treatment.

\subsection{Acknowledgement}

The authors would like to acknowledge the financial supports provided by Ministry of Higher Education Malaysia (HiCOE Grant: 4J182 and FRGS Grant: 4F920).

* Present address of C.S. Ong: Water desalination and Reuse Center, Division of Biological and Environmental Science and Engineering, King Abdullah University of Science and Technology, Thuwal 23955-6900, Saudi Arabia.

\section{Symbols Used}

\section{$\underline{\text { Symbols }}$}

$\Delta C_{\mathrm{f}}[\mathrm{g} / \mathrm{L}]$

change in salt concentration in feed solution

$\Delta P[\mathrm{bar}]$

applied pressure difference

$\Delta t[\mathrm{~min}]$

operating duration

$\Delta V[\mathrm{~mL}]$

change in volume of feed solution

$A \quad\left[\mathrm{~m}^{2}\right]$

effective area of membrane

$C_{\mathrm{f}}[\mathrm{S}]$

feed conductivity

$C_{\mathrm{p}}[\mathrm{S}]$

permeate conductivity

$J_{\mathrm{O}}\left[\mathrm{Lm}^{-2} \mathrm{~h}^{-1}\right]$

oil flux

$J_{\mathrm{O}, \mathrm{FO}} \quad\left[\mathrm{gm}^{-2} \mathrm{~h}^{-1}\right]$

oil flux FO mode

$J_{\mathrm{s}} \quad\left[\mathrm{Lm}^{-2} \mathrm{~h}^{-1}\right]$

salt flux 


$\begin{array}{lll}J_{\mathrm{v}} & {\left[\mathrm{Lm}^{-2} \mathrm{~h}^{-1}\right]} & \text { pure water flux } \\ R_{\mathrm{O}} & {[\%]} & \text { oil rejection } \\ R_{\mathrm{S}} & {[\%]} & \text { salt rejection } \\ V & {[\mathrm{~mL}]} & \text { volume of permeate } \\ X & {\left[\mathrm{Lm}^{-2} \mathrm{~h}^{-1} \mathrm{bar}^{-1}\right]} & \text { pure water permeability } \\ Y & {\left[\mathrm{Lm}^{-2} \mathrm{~h}^{-1} \mathrm{bar}^{-1}\right]} & \text { salt permeability coefficient }\end{array}$

\section{$\underline{\text { Abbreviations }}$}

AL-FS active layer facing feed solution orientation

Bpy dipyridyl

CNTs carbon nanotubes

$\mathrm{CuBr} \quad$ copper(I) bromide

DI deionized water

DMAEMA (dimethylamino)ethyl methacrylate

DS draw solution

EBiB ethyl 2-bromoisobutyrate

FO forward osmosis

$\mathrm{gMH} \quad \mathrm{gm}^{-2} \mathrm{~h}^{-1}$

GO graphene oxide

HMImCl 1-hexyl-3-methylimidazolium chloride

ICP Internal concentration polarization

IP interfacial polymerization

LMH $\quad \mathrm{Lm}^{-2} \mathrm{~h}^{-1}$

LMHB $\quad \mathrm{Lm}^{-2} \mathrm{~h}^{-1} \mathrm{Bar}^{-1}$

MPD m-Phenylenediamine

$\mathrm{NaCl}$ sodium chloride

NF nanofiltration

NMP N-methyl-2-pyrrolidone 
$\begin{array}{ll}\text { PA } & \text { polyamide } \\ \text { PES } & \text { polyethersulfone } \\ \text { PMAPS } & \text { poly[3-(N-2-methacryloylxyethyl-N,N-dimethyl)-ammonatopropanesulfonate] } \\ \text { PVP40 } & \text { polyvinylpyrrolidone40 } \\ \text { RO } & \text { reverse osmosis } \\ \text { SDS } & \text { sodium dodecyl sulfate } \\ \text { TFC } & \text { thin film composite } \\ \text { TMC } & \text { trimesoyl chloride } \\ \text { UF } & \text { ultrafiltration } \\ \text { Z-SWNT } & \text { zwitterionic single-walled nanotubes }\end{array}$

\section{Reference}

1. $\mathrm{Yu}, \mathrm{L} ., \mathrm{Han}, \mathrm{M}$. and He, F., "A review of treating oily wastewater", Arabian Journal of Chemistry, Vol., (2013).

2. Poulopoulos, S.G., Voutsas, E.C., Grigoropoulou, H.P. and Philippopoulos, C.J., "Stripping as a pretreatment process of industrial oily wastewater", Journal of Hazardous Materials, Vol. 117, (2005), 135-139.

3. Park, E. and Barnett, S.M., "Oil/water separation using nanofiltration membrane technology", Separation Science and Technology, Vol. 36, (2001), 1527-1542.

4. Kasemset, S., Lee, A., Miller, D.J., Freeman, B.D. and Sharma, M.M., "Effect of polydopamine deposition conditions on fouling resistance, physical properties, and permeation properties of reverse osmosis membranes in oil/water separation", Journal of Membrane Science, Vol. 425-426, (2013), 208-216.

5. Hickenbottom, K.L., Hancock, N.T., Hutchings, N.R., Appleton, E.W., Beaudry, E.G., Xu, P. and Cath, T.Y., "Forward osmosis treatment of drilling mud and fracturing wastewater from oil and gas operations", Desalination, Vol. 312, (2013), 60-66.

6. Duong, P.H.H., Chung, T.S., Wei, S. and Irish, L., "Highly permeable double-skinned forward osmosis membranes for anti-fouling in the emulsified oil-water separation process",

Environmental Science and Technology, Vol. 48, (2014), 4537-4545.

7. Mi, B. and Elimelech, M., "Organic fouling of forward osmosis membranes: Fouling reversibility and cleaning without chemical reagents", Journal of Membrane Science, Vol. $348,(2010), 337-345$.

8. Martinetti, C.R., Childress, A.E. and Cath, T.Y., "High recovery of concentrated RO brines using forward osmosis and membrane distillation", Journal of Membrane Science, Vol. 331, (2009), 31-39.

9. Song, X., Wang, L., Tang, C.Y., Wang, Z. and Gao, C., "Fabrication of carbon nanotubes incorporated double-skinned thin film nanocomposite membranes for enhanced separation performance and antifouling capability in forward osmosis process", Desalination, Vol. 369, (2015), 1-9. 
10. Zhang, S., Wang, P., Fu, X. and Chung, T.-S., "Sustainable water recovery from oily wastewater via forward osmosis-membrane distillation (FO-MD)", Water Research, Vol. 52, (2014), 112-121.

11. Lau, W.J., Gray, S., Matsuura, T., Emadzadeh, D., Chen, J.P. and Ismail, A.F., "A review on polyamide thin film nanocomposite (TFN) membranes: History, applications, challenges and approaches", Water Res, Vol. 80, (2015), 306-24.

12. Lv, L., Xu, J., Shan, B. and Gao, C., "Concentration performance and cleaning strategy for controlling membrane fouling during forward osmosis concentration of actual oily wastewater", Journal of Membrane Science, Vol. 523, (2017), 15-23.

13. Li, P., Lim, S.S., Neo, J.G., Ong, R.C., Weber, M., Staudt, C., Widjojo, N., Maletzko, C. and Chung, T.S., "Short- and long-term performance of the thin-film composite forward osmosis (TFC-FO) hollow fiber membranes for oily wastewater purification", Industrial and Engineering Chemistry Research, Vol. 53, (2014), 14056-14064.

14. Han, G., de Wit, J.S. and Chung, T.-S., "Water reclamation from emulsified oily wastewater via effective forward osmosis hollow fiber membranes under the PRO mode", Water Research, Vol. 81, (2015), 54-63.

15. Ong, R.C., Chung, T.-S., Helmer, B.J. and de Wit, J.S., "Characteristics of water and salt transport, free volume and their relationship with the functional groups of novel cellulose esters", Polymer, Vol. 54, (2013), 4560-4569.

16. Tiraferri, A., Yip, N.Y., Phillip, W.A., Schiffman, J.D. and Elimelech, M., "Relating performance of thin-film composite forward osmosis membranes to support layer formation and structure", Journal of Membrane Science, Vol. 367, (2011), 340-352.

17. Chan, W.-F., Marand, E. and Martin, S.M., "Novel zwitterion functionalized carbon nanotube nanocomposite membranes for improved RO performance and surface anti-biofouling resistance", Journal of Membrane Science, Vol. 509, (2016), 125-137.

18. Chan, W.F., Chen, H.Y., Surapathi, A., Taylor, M.G., Shao, X., Marand, E. and Johnson, J.K., "Zwitterion functionalized carbon nanotube/polyamide nanocomposite membranes for water desalination", ACS Nano, Vol. 7, (2013), 5308-19.

19. Kobayashi, M., Terayama, Y., Kikuchi, M. and Takahara, A., "Chain dimensions and surface characterization of superhydrophilic polymer brushes with zwitterion side groups", Soft Matter, Vol. 9, (2013), 5138.

20. Ong, C.S., Al-anzi, B., Lau, W.J., Goh, P.S., Lai, G.S., Ismail, A.F. and Ong, Y.S., "Anti-Fouling Double-Skinned Forward Osmosis Membrane with Zwitterionic Brush for Oily Wastewater Treatment", Scientific Reports, Vol. 7, (2017), 6904.

21. Chun, Y., Kim, S.-J., Millar, G.J., Mulcahy, D., Kim, I.S. and Zou, L., "Forward osmosis as a pretreatment for treating coal seam gas associated water: Flux and fouling behaviour", Desalination, Vol. 403, (2017), 144-152.

22. Zhu, Y., Zhang, F., Wang, D., Pei, X.F., Zhang, W. and Jin, J., "A novel zwitterionic polyelectrolyte grafted PVDF membrane for thoroughly separating oil from water with ultrahigh efficiency", Journal of Materials Chemistry A, Vol. 1, (2013), 5758-5765.

23. Schlenoff, J.B., "Zwitteration: Coating Surfaces with Zwitterionic Functionality to Reduce Nonspecific Adsorption", Langmuir, Vol. 30, (2014), 9625-9636.

24. Vatanpour, V. and Zoqi, N., "Surface modification of commercial seawater reverse osmosis membranes by grafting of hydrophilic monomer blended with carboxylated multiwalled carbon nanotubes", Applied Surface Science, Vol. 396, (2017), 1478-1489.

25. Wei, J., Qiu, C., Tang, C.Y., Wang, R. and Fane, A.G., "Synthesis and characterization of flatsheet thin film composite forward osmosis membranes", Journal of Membrane Science, Vol. 372, (2011), 292-302.

26. Offeman, R.D. and Ludvik, C.N., "A novel method to fabricate high permeance, high selectivity thin-film composite membranes", Journal of Membrane Science, Vol. 380, (2011), 163-170. 
27. Xu, Y., Peng, X., Tang, C.Y., Fu, Q.S. and Nie, S., "Effect of draw solution concentration and operating conditions on forward osmosis and pressure retarded osmosis performance in a spiral wound module", Journal of Membrane Science, Vol. 348, (2010), 298-309.

28. You, M., Wang, P., Xu, M., Yuan, T. and Meng, J., "Fouling resistance and cleaning efficiency of stimuli-responsive reverse osmosis (RO) membranes", Polymer, Vol. 103, (2016), 457-467.

29. Zhang, X., Tian, J., Gao, S., Shi, W., Zhang, Z., Cui, F., Zhang, S., Guo, S., Yang, X., Xie, H. and Liu, D., "Surface functionalization of TFC FO membranes with zwitterionic polymers: Improvement of antifouling and salt-responsive cleaning properties", Journal of Membrane Science, Vol. 544, (2017), 368-377. 